\title{
An Efficient Arrhythmia Detection Using Autocorrelation and Statistical Approach
}

\author{
Mohamad Sabri Bin Sinal, Eiji Kamioka \\ Graduate School of Engineering and Science, Shibaura Institute of Technology, Tokyo, Japan \\ Email: nb16109@shibaura-it.ac.jp,kamioka@shibaura-it.ac.jp
}

How to cite this paper: Sinal, M.S.B. and Kamioka, E. (2018) An Efficient Arrhythmia Detection Using Autocorrelation and Statistical Approach. Journal of Computer and Communications, 6, 63-81. https://doi.org/10.4236/jcc.2018.610006

Received: September 9, 2018

Accepted: October 28, 2018

Published: October 31, 2018

Copyright $\odot 2018$ by authors and Scientific Research Publishing Inc. This work is licensed under the Creative Commons Attribution International License (CC BY 4.0).

http://creativecommons.org/licenses/by/4.0/

\section{cc) (i) Open Access}

\begin{abstract}
Computational electrocardiogram (ECG) analysis is one of the most crucial topics in cardiovascular research domain especially in identifying abnormalities of heart condition through cardiac arrhythmia symptom. There are many existing works focusing on recognizing the abnormalities condition through arrhythmia symptom, however, the detection rate is still unsatisfied. Arrhythmia consists of more than 14 various types of symptoms. Therefore, most of the existing research found it difficult to classify the entire symptom and maintain the overall accuracy especially in long hour data. In this study, a new mechanism to overcome this issue is proposed: A combination between Autocorrelation methods with K-Nearest Neighbor (KNN) classifier method is introduced to accurately and robustly detect 14 types of Arrhythmia symptom regardless of the origin of the symptom in a long hour data. Moreover, variability analysis based on periodic autocorrelation result is proposed and used for classification procedure. 1 minute and 12 hours duration data was chosen to compare and signify the most suitable time duration to detect Arrhythmia symptom. In addition, an analytical result and discussion is done to provide justification behind each tendency of Arrhythmia and Normal Sinus symptom in autocorrelation result. As the result of proposed method performance evaluation, it was revealed that the accuracy of $95.5 \%$ in discriminating Arrhythmia from Normal Sinus data is achieved. Furthermore, it was confirmed that utilizing autocorrelation result in long hour data can help to generalize abnormalities characteristic of heart condition like Arrhythmia symptom. It is concluded that the proposed method can be useful to diagnose abnormalities of heart condition at any stage.
\end{abstract}

\section{Keywords}

Autocorrelation Analysis, Arrhythmia Diagnosis, KNN Classifier, Autocorrelation Function, Heart Disease Diagnosis, Computational Heart Disease Diagnosis 


\section{Introduction}

Electrocardiography (ECG) is a recording process of the heart electrical activities over a period of time. It is a non-invasive examination that is used to diagnose underlying heart conditions using electrodes placed on the skin. The analysis of the electrocardiographic signals provides detailed information on cardiac health status. There are various types of irregular heart rhythms associated with characteristic electrocardiogram (ECG) patterns. One of the types is Cardiac Arrhythmia. Cardiac Arrhythmia is a group of conditions in which the heartbeat is irregularly faster or slower than normal conditions. Cardiac Arrhythmia is classified into two major categories. One is Morphological Arrhythmia formed by a single irregular heartbeat. The other is Rhythmic Arrhythmia formed by a set of irregular heartbeats. The ECG data including Cardiac Arrhythmia symptoms is characterized by the disappearance of $\mathrm{P}$ wave, by irregular $\mathrm{R}$ to $\mathrm{R}$ interval or by fast heart rate (e.g. 250 - 400 beats per minute). Cardiac Arrhythmia is accompanied with a various degrees of atrial ventricular [1].

The procedure to detect Arrhythmia symptoms is complicated since the analysis of heartbeats of ECG data requires Holter monitor for hours or even for a day. Normally, ECG data may include various types of human errors and measurement errors. Therefore, it is challenging to identify Arrhythmia symptoms when signal noise and disease symptoms are mixed in one data. As an alternative solution to overcome this issue, a computational based approach is proposed in this paper.

Diagnosis of cardiac abnormalities from ECG signals has been proposed by many researchers. Conventionally, an automatic heart disease classification approach consists of 4 processes, which are: 1) ECG signal processing, 2) heartbeat segmentation, 3) feature extraction, and 4) classification. The main conventional features used in the feature extraction process are higher order statistical feature [2] [3], morphological features [4] [5] [6], independent component analysis and wavelet features [7] [8] [9] [10]. In the classification process, artificial neural network [7] [11], linear discrimination analysis (LDA), ensemble method [6], support vector machine [3] [8] [10] and self-organizing map are commonly used in this research area. However, there are two concerns for this study. The first one is the feature extraction process to extract Arrhythmia and abnormalities symptom in long hour data. Second one is the mechanism to analyze and classify the disease itself. Considering the mechanism of Arrhythmia, the symptoms seen in ECG data can be divided into two segments in heart cycle, which are atrial segment and ventricular segment. Most of the works take into consideration either abnormality in atrial segment or in ventricular segment of ECG signals [12] [13] [14] [15] [16]. Since Arrhythmia is the most well-known symptom leading to the sudden cardiac arrest (SCA), automatic cardiac diagnosis systems should have a property to detect the maximum number of abnormalities irrespective of the origin. Moreover, the symptoms randomly appear over a period of time, hence, ECG analysis focusing on only a small period of time results in an insuffi- 
cient and inaccurate diagnosis. Additionally, automatic disease classification systems should have a capability to classify the abnormalities from the normal condition with high accuracy, less complexity and low computational cost.

To overcome the issues addressed above, in this paper, a new mechanism, which accurately and robustly detects Arrhythmia symptoms from 12 hours ECG data regardless the origin of Arrhythmia symptoms, will be proposed. The new mechanism can mainly be divided into two steps. First, an autocorrelation technique is used to investigate the cyclic nature of a time series ECG data. The result provides the abnormalities in terms of periodicity. Moreover, it computationally simplifies the abnormalities detection process which enhances the detection efficiency. Second, Arrhythmia symptoms are accurately detected based on the features of autocorrelation result such as cyclic peak value and its time length of the first slope. This achieves the classification of Arrhythmia symptoms from Normal Sinus rhythm quantitatively with K-Nearest Neighbors (KNN). Note that Normal Sinus rhythm represents the characteristic of healthy human heart condition and it is used as a reference to identify the Arrhythmia symptoms.

The remainder of this paper is organized as follows: Section 2 will introduce Normal Sinus and Arrhythmia Rhythms, showing their characteristic wave-forms in ECG data. Section 3 will discuss the related works to clarify the issues of existing methods in detecting heart cycle and Arrhythmia symptoms in ECG data. Section 4 will describe the methodology of the proposed mechanism which elaborately detects characteristic wave-forms in ECG data and identifies the Arrhythmia. In Section 5, the evaluation result of the proposed mechanism will be shown based on the two techniques, autocorrelation and statistical approach. In Section 6, the conclusion will be made with discussions on possible future study.

\section{Principle of Electrocardiogram}

Electrocardiography is considered the most conventional procedure to measure heart condition. It records the heart's electrical activities in time domain to check the abnormalities that may exist. It also provides information of the patient's heart rate and rhythm and shows if there is enlargement in the heart due to diseases such as high blood pressure (hypertension) or evidence of a previous heart attack (myocardial infarction). Once all the analysis is done, a proper treatment can be made accordingly based on the findings. A typical ECG waveform periodically consists of 5 main waves; $\mathrm{P}, \mathrm{Q}, \mathrm{R}, \mathrm{S}$ and $\mathrm{T}$ waves. The $\mathrm{P}$ wave represents the depolarization of the right and left atria. The $\mathrm{P}, \mathrm{Q}, \mathrm{R}$ and the $\mathrm{S}$ waves depict the activation of the right and left ventricles, while the $\mathrm{T}$ wave shows the repolarization of the ventricular. It is indispensable to detect periodic heart cycle in order to identify any disease inside the heart. In this paper, two types of symptoms are mainly focused on which are Normal Sinus and Arrhythmia. 


\subsection{Normal Sinus}

Sinus rhythm is the set of heart's normal regular rhythms where the pacemaker is in the sinoatrial node and the conduction is going through the atria ventricular node, and the ventricles are unimpaired. It is a reflection of a normally functioning conduction system in the body. The electrical current is following in the normal conduction pathway without interference from other factors such as bodily system or disease processes [17].

\subsection{Arrhythmia}

Arrhythmia refers to any irregular change from the normal sequence of electrical impulses of the heart, that is to say, the electrical impulses could be too fast, too slowly or erratic. If the heartbeat is too fast, it is called tachycardia, while the heartbeat is too slow, it is called bradycardia. Arrhythmia consists of more than 10 various types of abnormality symptoms. For each symptom, it consists of its own unique identification characterization.

\subsection{P, Q, R, S, T Wave Morphology}

Atrial and ventricular depolarization and repolarization are represented on the ECG as a series of waves: $\mathrm{P}$ wave is followed by QRS complex and T wave. The first deflection is the $\mathrm{P}$ wave which is associated with the right and the left atrial depolarization. The second wave is the QRS complex. By convention, if the first deflection in the complex is negative, it is called $\mathrm{Q}$ wave. The first deflection in the complex is positive, it is called $\mathrm{R}$ wave. A negative deflection after the $\mathrm{R}$ wave is called $\mathrm{S}$ wave. The $\mathrm{T}$ wave represents ventricular repolarization. The polarity of this wave normally follows that of the main QRS deflection. The ventricles are electrically unstable during the period of repolarization extending from the peak of the $\mathrm{T}$ wave to its initial downslope. A normal ECG signal is considered as a periodic signal. If there are irregularities in these waves, they could be signs of a heart problem. Figure 1 and Figure 2 represent Normal Sinus and Arrhythmia waveforms, respectively. Both waveforms show a huge difference in many ways as shown in Figure 1 and Figure 2. In this research, the behavior within the range of a heart cycle and the interval between each heart cycles are the main focuses. The analysis of autocorrelation result to identify and classify the diseases is the main concern for this study.

\section{Related Works}

Uday Maji et al. [12] proposed an ECG signal analysis by using Variational Mode Decomposition (VMD). This method is used to characterize atrial and ventricular Arrhythmia simultaneously and independently from each heart cycle segments. VMD is a non-recursive signal decomposition method proposed by Dragomiretskiy et al. It is able to decompose the input signal into desired number of mode N. The experimental result revealed that the Arrhythmia detection accuracy of $99.1 \%$ and $99.8 \%$ at ventricular segment and at atrial segment, 


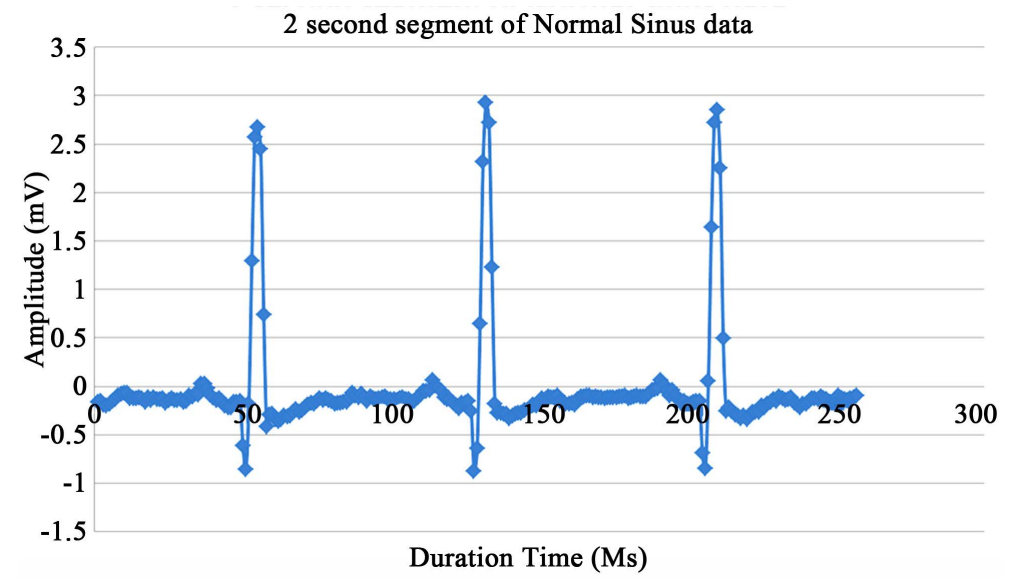

Figure 1. ECG data with normal sinus symptoms.

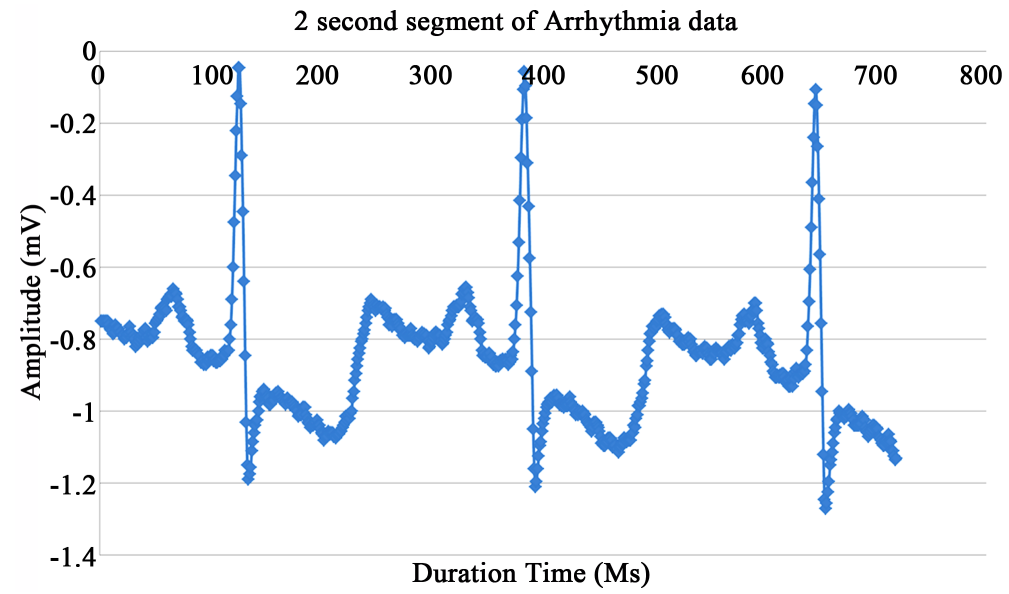

Figure 2. ECG data with arrhythmia symptoms.

respectively, has been achieved. However, this method relies on two class multi stage classifications. The complexities in classification become the main disadvantage for this study because it requires to separate the ECG segment into two parts for further step in abnormalities analysis. Therefore, this characterization of abnormalities under Arrhythmia symptom is less efficient and may consume more computational cost to reach the purpose.

Rekha Rajagopal et al. [18] review the performance of five different types of unsupervised dimensionality reduction (DR) techniques in classifying cardiac Arrhythmias. DR technique is a method representing high-dimensional data in lower-dimensional spaces. Since interpretation of high-dimensional data remains a difficult task, DR technique is considered one of the best alternative solutions to overcome the issue. In this study, they include principal component analysis (PCA), fast independent component analysis (fast ICA) with tangential, kurtosis and Gaussian contrast function, Kernel PCA (KPCA) with polynomial kernel, hierarchical nonlinear PCA (hNLPCA) and principal polynomial analysis (PPA) for comparative study. The overall result demonstrates that the combination of probabilistic Neural Networks (PNN) classifier and fast ICA DR tech- 
nique with tangential contrast function shows a high accuracy of $99.3 \%$ to classify Arrhythmia. However, most of these approaches are mainly required a huge amount of time even for low dimensional mapping. Moreover, most of them in this study are complex in terms of implementation. Therefore, in this paper, it is expected that the number of parameters should be minimal with a high accuracy in detecting Arrhythmia symptoms.

Likewise, Chia et al. [19] proposed hybrid adaptive feature selection mechanism for detection of Arrhythmia in ECG data. A combination of k-mean clustering and support vector machine was introduced and tested with more than 100,000 samples of Arrhythmia symptoms. The accuracy of $98.92 \%$ was achieved to detect Arrhythmia. However, the accuracy of the proposed method is relies on three feature extraction processes such as screening feature sample, partitioning the right sample and lastly balancing the number of sample before the classification in order to maintain a high detection rate. Most of the existing researches have shown that the characterization of abnormalities through Arrhythmia symptoms is very complex even with a computational method. The degree of complexity can be seen from the initial stage of preparing the sample feature until characterizing the abnormalities itself. Therefore, an autonomous and simple approach with a high detection accuracy, which adaptively identifies various types of abnormalities of heart condition through Arrhythmia symptoms, will be the main focus for this study.

\section{Detection of Arrhythmia Symptoms Using Autocorrelation and KNN Classifier}

In this section, an autocorrelation-based approach with KNN classifier is proposed to classify the Arrhythmia from Normal Sinus. Autocorrelation is a statistical method that can measure internal correlation within a time series domain. It is defined based on the concept of time lag. Performing autocorrelation of a time series data is beneficial especially to identify signal stationary condition, measure variability level of continuous data or even indicate quantitative relation of some previous data points occurring with a time lag. Conventionally, ECG data is a time series data and it provides very useful information of heart condition. There are various types of heart disease symptoms and the characteristic differs from one symptom to another. However, there is a common characteristic shared among heart disease symptoms, which is inconsistent irregularity in shape that appears in time series domain. In this study, a quantitative analysis is proposed to numerically characterize the two symptoms. Variability analysis based on the first autocorrelation periodic cyclic result is introduced. Two parameters, which come from the first periodic slope of autocorrelation result, will be used for analysis. Based on the two parameters, a classification procedure to discriminate the two different groups of data will be performed by using KNN classifier. KNN is a common classification method used in pattern recognition. Without relying on any specific segment in ECG cycle to classify the disease accurately, an autocorrelation function can simplify the detection me- 
chanism by relying on variability behavior of large group data from two symptoms only. The details of procedure for autocorrelation, $\mathrm{KNN}$ and the analysis will be stated in Sections 4.1 and 4.2.

\subsection{Autocorrelation Coefficient}

Autocorrelation also known as "serial correlation" or "lagged correlation" is a statistical method that measures dependency of variables arranged in time. There are three tools for assessing the autocorrelation of a time series data which are time series plot, the lagged scatter plot and the autocorrelation function. In this study, autocorrelation function will be used to measure variability level of long hour serial correlation of ECG data. Here in this section, an autocorrelation coefficient function will be explained in detail.

Let $y_{i}(i=1, n)$ be a time series data at the data point $i$, and let $\alpha$ be the average value of all the data.

The autocorrelation function at lag $k$ is defined as:

$$
r_{k}=\frac{\sum_{i=1}^{n-k}\left(y_{i}-\alpha\right)\left(y_{i+k}-\alpha\right)}{\sum_{i=1}^{n}\left(y_{i}-\alpha\right)^{2}}
$$

As the autocorrelation function shows, $r_{\mathrm{k}}$ describes the correlation between the two data which are located with the lag $k$ each other. The score ranges from -1.0 (perfect negative relation) to +1.0 (perfect positive relation). If there is no correlation between the two variables, the score is zero.

Next, the procedure to classify the two symptoms is mentioned below and shown in Figure 3.

1) Perform an autocorrelation to 12 hours ECG data and describe the score with lag $k$ as the autocorrelation result.

2) Investigate the first periodic slope segment in autocorrelation result based on two parameters.

The two parameters are:

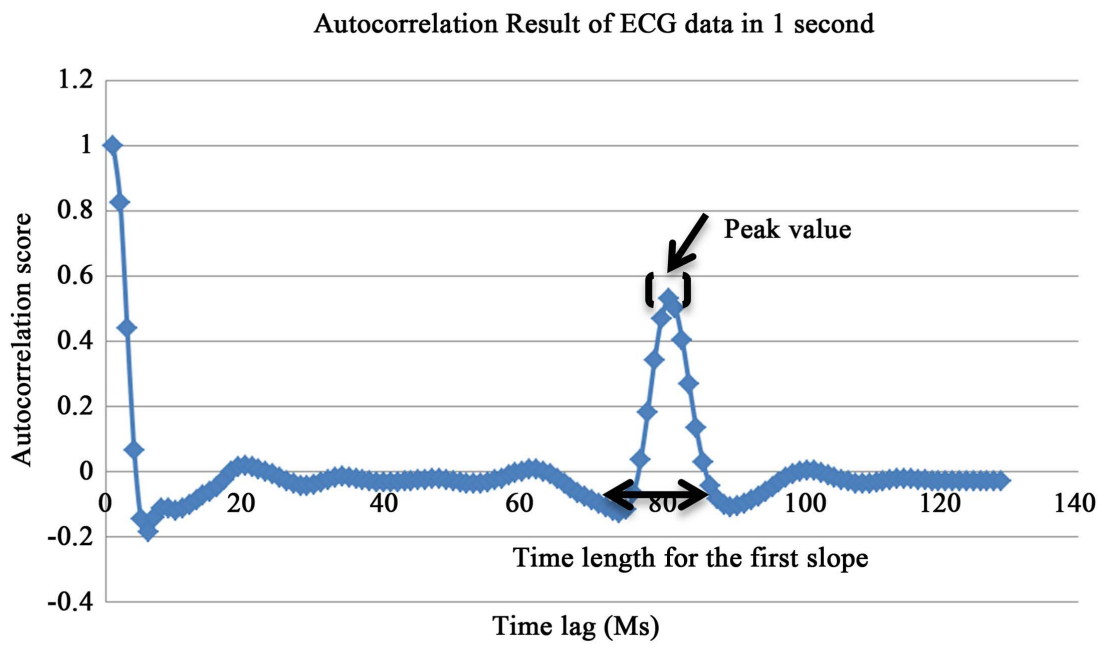

Figure 3. Proposed automatic mechanism to detect arrhythmia symptom. 
a) Peak values of the first periodic slope.

b) Time length of the first periodic slope as shown in Figure 4 .

Figure 5 represent 2 second segment of Normal Sinus autocorrelation result and Figure 6 represent 2 second segment of Arrhythmia autocorrelation result in 1 minute duration. For Figure 7, it represent 2 second segment of Normal Sinus autocorrelation result in 12 hours duration while Figure 8 represent Arrhythmia autocorrelation result with the same duration. In these figures, the horizontal axis indicates the time lag of time series data while the vertical axis indicates the autocorrelation score of serial correlation output. Note that the first periodic slope peak value of autocorrelation result for Normal Sinus is much lower than the one for Arrhythmia.

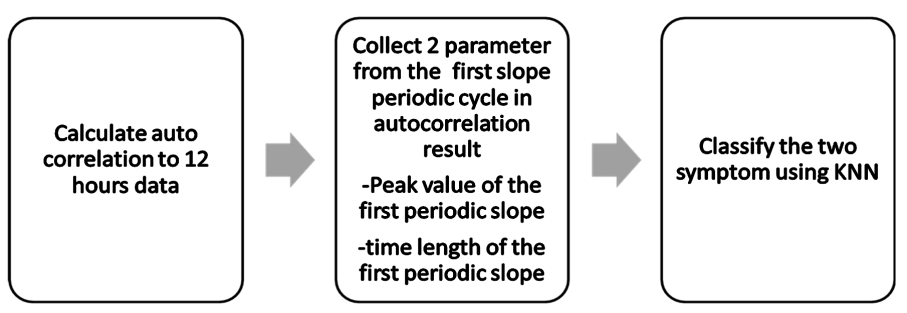

Figure 4. Two important parameters for classification at the first periodic slope.

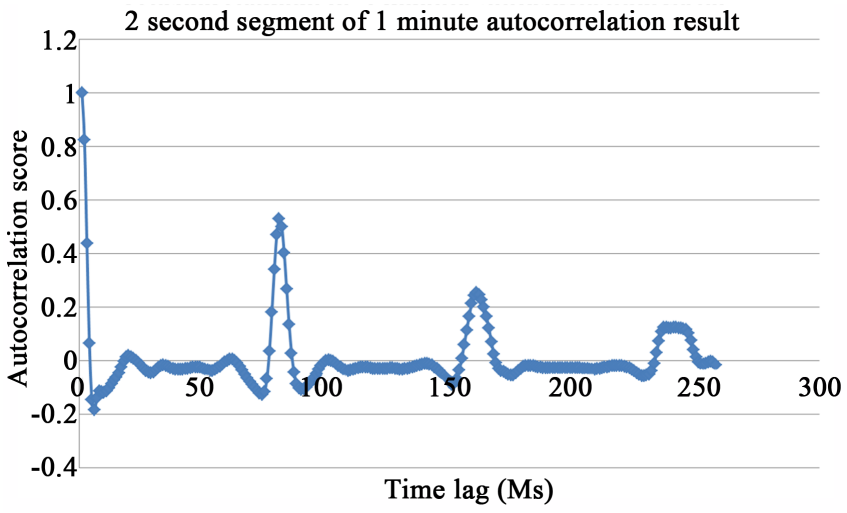

Figure 5. Example of 2 second segment in 1 minute autocorrelation result with normal sinus symptoms.

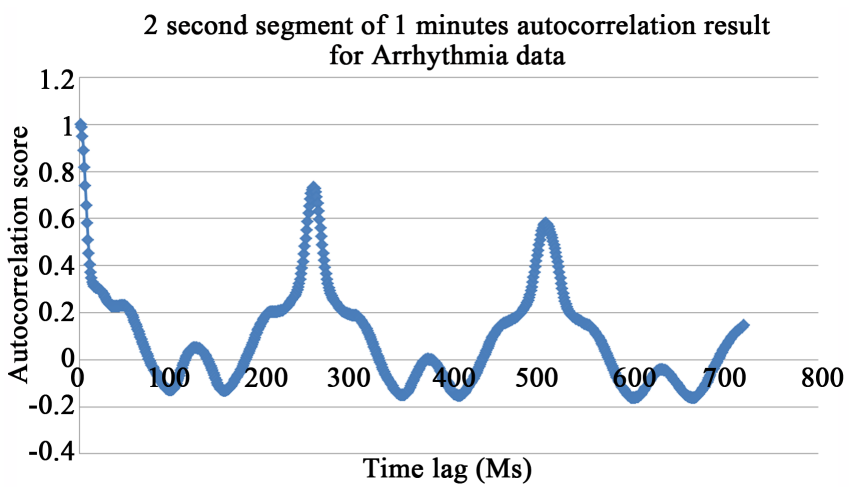

Figure 6. Example of 2 second segment in 1 minute autocorrelation result with arrhythmia symptoms. 


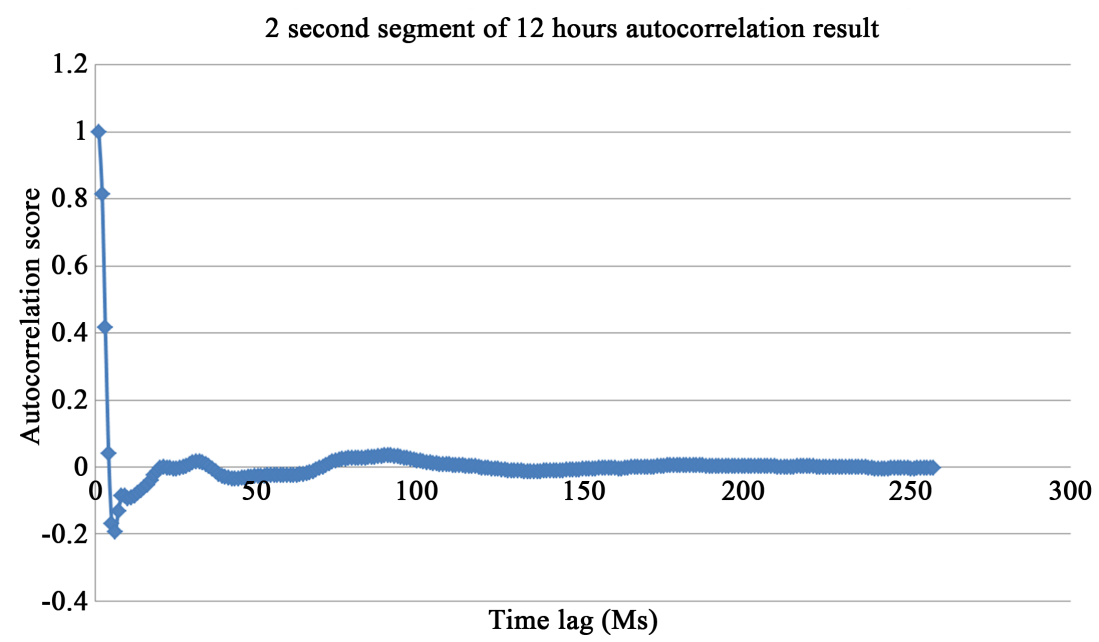

Figure 7. Example of 2 second segment in 12 hours autocorrelation result with normal sinus symptoms.

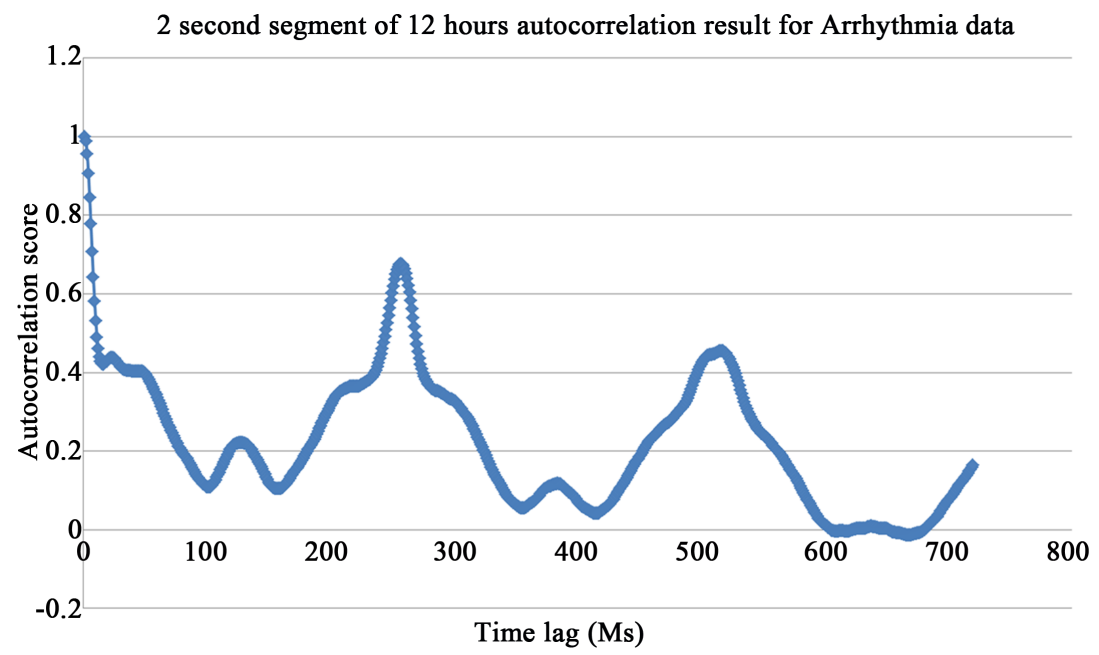

Figure 8. Example of 2 second segment in 12 hours autocorrelation result with arrhythmia symptoms.

\subsection{Discrimination of Arrhythmia from Normal Sinus with Supervised Machine Learning Classifier}

In this research, the values of significant first peak and time length of each first periodic slope in autocorrelation result are investigated in detail. KNN classifier is introduced to discriminate Arrhythmia symptoms from Normal Sinus. KNN is an instance-based learning method which keeps all available cases and classifies new cases based on a similarity measurement using distance function. KNN is a non-parametric approach and performs very fast. Also it makes no assumption about the data distribution before classification. As a result, the flexibility of KNN's decision boundary is a huge advantage compared to other approaches which rely on linear, elliptic or parabolic decision boundaries to classify specific group of data. Therefore, this method has been chosen for this study to classify the Arrhythmia symptoms from Normal Sinus. 


\section{Performance Evaluation}

In this section, the performance of the proposed method to discriminate Arrhythmia symptoms from Normal Sinus symptom is evaluated. In the following subsections, the database used for the evaluation experiment and the analytical results are discussed.

\subsection{Database for Evaluation}

Two types of database were introduced in this study, which are "MIT-BIH Arrhythmia" and "MIT-BIH Normal Sinus". They have been provided by Physionet [20]. Physionet is an open source database which provided a large number of recorded physiological data and the related open-source software. 48 records from MIT-BIH Arrhythmia and 17 records from MIT-BIH Normal Sinus were selected to evaluate the performance of the proposed mechanism. MIT-BIH Arrhythmia includes 14 types of abnormalities as shown in Table 1. The sampling frequency for each record is $360 \mathrm{~Hz}$. MIT-BIH Normal Sinus consists of ECG data with healthy heart condition. The sampling frequency for each record is $129 \mathrm{~Hz}$. The amplitude range of typical ECG signal is from $-5 \mathrm{mV}$ to $5 \mathrm{mV}$. The time duration for each record is approximately 12 hours.

Table 1. Detail of information for MIT-BIH arrhythmia database.

\begin{tabular}{|c|c|}
\hline \multicolumn{2}{|c|}{ MIT-BIH Arrhythmias Database Detail Information } \\
\hline Heartbeat Type & Total \\
\hline Normal rhythm & 74,607 \\
\hline Left bundle branch block & 8069 \\
\hline Right bundle branch block & 7250 \\
\hline Atrial premature contraction & 2514 \\
\hline Premature ventricular contraction & 7127 \\
\hline Paced beat & 7020 \\
\hline Aberrated atrial premature beat & 150 \\
\hline Ventricular flutter wave & 472 \\
\hline Fusion of ventricular and normal beat & 802 \\
\hline Non-conducted P-wave (Blocked APC) & 193 \\
\hline Nodal (Junctional) escape beat & 229 \\
\hline Fusion of paced and normal beat & 986 \\
\hline Ventricular escape beat & 106 \\
\hline Nodal (Junctional) escape beat & 83 \\
\hline Atrial escape beat & 16 \\
\hline Unclassified beat & 35 \\
\hline Total & 109,655 \\
\hline
\end{tabular}


As mention in Section 1, the autocorrelation was performed for all the ECG data; MIT-BIH Arrhythmia and MIT-BIH Normal Sinus, selected from the abovementioned database. The input parameters for KNN classifier were the autocorrelation score and the time length of the first periodic slope for each ECG data. Since there are various types of Arrhythmia symptoms covered in this research, the effectiveness of the proposed mechanism was evaluated based on the capability to detect the symptom regardless the origin and classifying accuracy.

\subsection{Result of Experiment}

Quantitative analyses were performed to evaluate the proposed method. To demonstrate the performance improvement with the proposed Arrhythmia detection mechanism, the performance evaluation was divided into two sections: 1) Accuracy, sensitivity and specificity of the proposed method to discriminated Arrhythmia from Normal Sinus, 2) Statistical analysis of the proposed approach.

\section{Accuracy, Sensitivity and Specificity Evaluation}

To evaluate the classification performances of the proposed mechanism, "Accuracy", "Sensitivity" and "Specificity" were selected as the evaluation metrics. The sensitivity and specificity are considered as the best paired performance metrics to evaluate the classification accuracy of heart disease [21]. The true positive rate of sensitivity represents the proportion of actual positives correctly identified as Arrhythmia data as having the Arrhythmia [22]. On the other hand, the true negative rate of specificity represents the proportion of actual negatives correctly identified as Normal Sinus and not having the Arrhythmia condition [22]. Accuracy rate represent the overall ratio of the proposed method to differentiate Normal Sinus and Arrhythmia correctly [22]. The discrimination accuracy, sensitivity and specificity are defined as follows:

$$
\begin{aligned}
& \quad \text { Sensitivity }=\text { True positives } / \text { True positive }+ \text { False negative }) \\
& \text { Specificity }=\text { True negatives } /(\text { True negative }+ \text { False positives }) \\
& \text { Accuracy }=(\text { True negatives }+ \text { True positive }) /(\text { True negatives }+ \text { True positive } \\
& + \text { False negative }+ \text { False positive }) \\
& \text { where, }
\end{aligned}
$$

True positive: The number of Arrhythmia data correctly identified as Arrhythmia data.

False negative. The number of Arrhythmia data incorrectly identified as NS data.

False positive: The number of NS data incorrectly identified as Arrhythmia data.

True negative: The number of NS data correctly identified as NS data.

Figure 9 and Figure 10 represent the distribution of the peak value and the time length of the first periodic slope with 66 patients' data with 1 minute and 12 hours duration, respectively. The result in Figure 9 shows that the Arrhythmia data and Normal Sinus data are not well discriminated. On the other hand, the result in Figure 10 shows that almost all the Arrhythmia data is scattered 


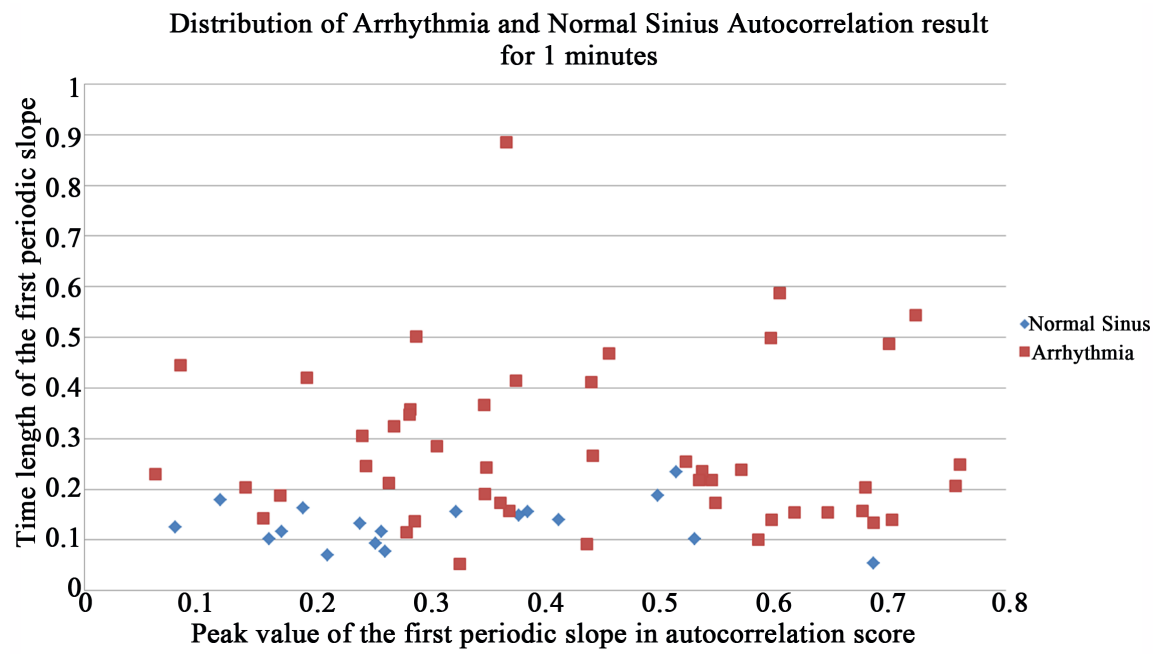

Figure 9. Distribution of autocorrelation result based on first periodic slope peak value with time length for 1 minute data.

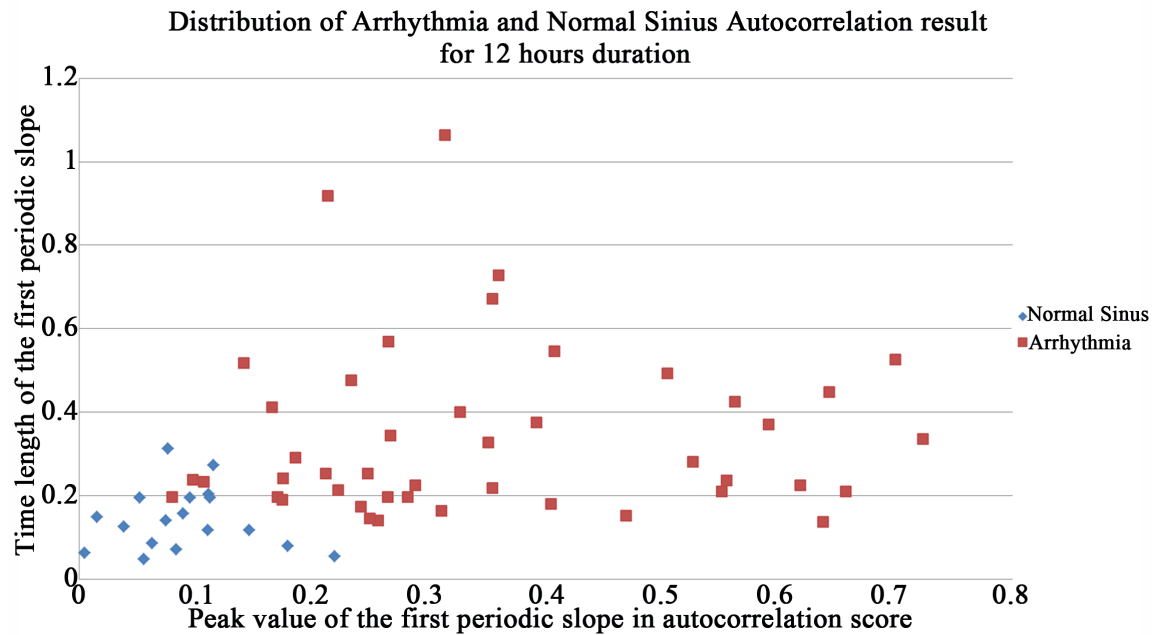

Figure 10. Distribution of autocorrelation result based on first periodic slope peak value with time length for 12 hours data.

without overlapping the Normal Sinus data. Only three Arrhythmia data are overlapped with the region of Normal Sinus data. In order to classify these two symptoms computationally, a KNN classifier was used.

In this evaluation, to classify Arrhythmia from Normal Sinus, KNN is used. 83.3\% sensitivity of Arrhythmia detection and 55.5\% specificity of Normal Sinus detection were achieved for 1 minute's duration data. However, $97.9 \%$ sensitivity of Arrhythmia detection and $88.8 \%$ specificity of Normal Sinus detection were achieved for 12 hours duration data as shown in Table 2. The higher rate of Arrhythmia detection was achieved even all 14 various types of symptoms were normalized into a series of correlation using autocorrelation. It is confirmed that the peak value and the time length of the first periodic slope in the autocorrelation result for 12 hours duration data are effective parameters to classify Arrhythmia from Normal Sinus with high detection accuracy. 
In order to compare the KNN classifier used here with others in the proposed method, other 17 types of supervised machine learning classifiers were selected and evaluated. Table 3 represent the performance comparison using 17 type of classifier using same dataset with 1 minute duration while Table 4 is focusing on the performance comparison using 17 type of classifier with 12 hours duration. Figure 11 and Figure 12 represent the overall performance of 1 minute and 12 hours data based on 17 various types of classifier in histogram plot. The evaluation metrics for the performance comparison are accuracy, sensitivity and specificity.

Table 2. Sensitivity, specificity and accuracy of Arrhythmia and normal sinus symptom detection using $\mathrm{KNN}$ with the proposed method.

\begin{tabular}{ccc}
\hline \multicolumn{3}{c}{ Performance of proposed method (Fine KNN + Autocorrelation) } \\
\hline & 1 minute & 12 hours \\
\hline Accuracy & $72.7 \%$ & $95.5 \%$ \\
Sensitivity & $83.3 \%$ & $97.9 \%$ \\
Specificity & $55.5 \%$ & $88.8 \%$ \\
\hline
\end{tabular}

Table 3. Performance comparison using 17 types of classifiers using the same dataset with 1 minute duration.

\begin{tabular}{|c|c|c|c|}
\hline \multicolumn{4}{|c|}{ Overall performance of 17 various classifier } \\
\hline Classifier Name Accuracy (\%) & & Sensitivity (\%) & Specificity (\%) \\
\hline Linear SVM & 78.8 & 89.5 & 50 \\
\hline Quadratic SVM & 75.8 & 85.4 & 55.5 \\
\hline Cubic SVM & 80.3 & 91.6 & 72.2 \\
\hline Fine Gaussian SVM & 78.8 & 77.0 & 55.5 \\
\hline Medium Gaussian SVM & 75.8 & 85.4 & 50 \\
\hline Coarse Gaussian SVM & 72.7 & 100 & 0 \\
\hline Fine KNN (Proposed method) & 72.7 & 83.3 & 55.5 \\
\hline Medium KNN & 81.8 & 89.5 & 61.1 \\
\hline Coarse KNN & 72.7 & 100 & 0 \\
\hline Cosine KNN & 74.2 & 83.3 & 50 \\
\hline Cubic KNN & 83.3 & 89.5 & 66.6 \\
\hline Weighted KNN & 78.8 & 87.5 & 55.5 \\
\hline Linear Discrimination & 78.8 & 91.6 & 44.4 \\
\hline Quadratic Discrimination & 81.8 & 83.3 & 77.7 \\
\hline Complex Tree & 80.3 & 81.25 & 77.7 \\
\hline Medium Tree & 80.3 & 81.25 & 77.7 \\
\hline Simple Tree & 78.8 & 83.3 & 66.6 \\
\hline
\end{tabular}




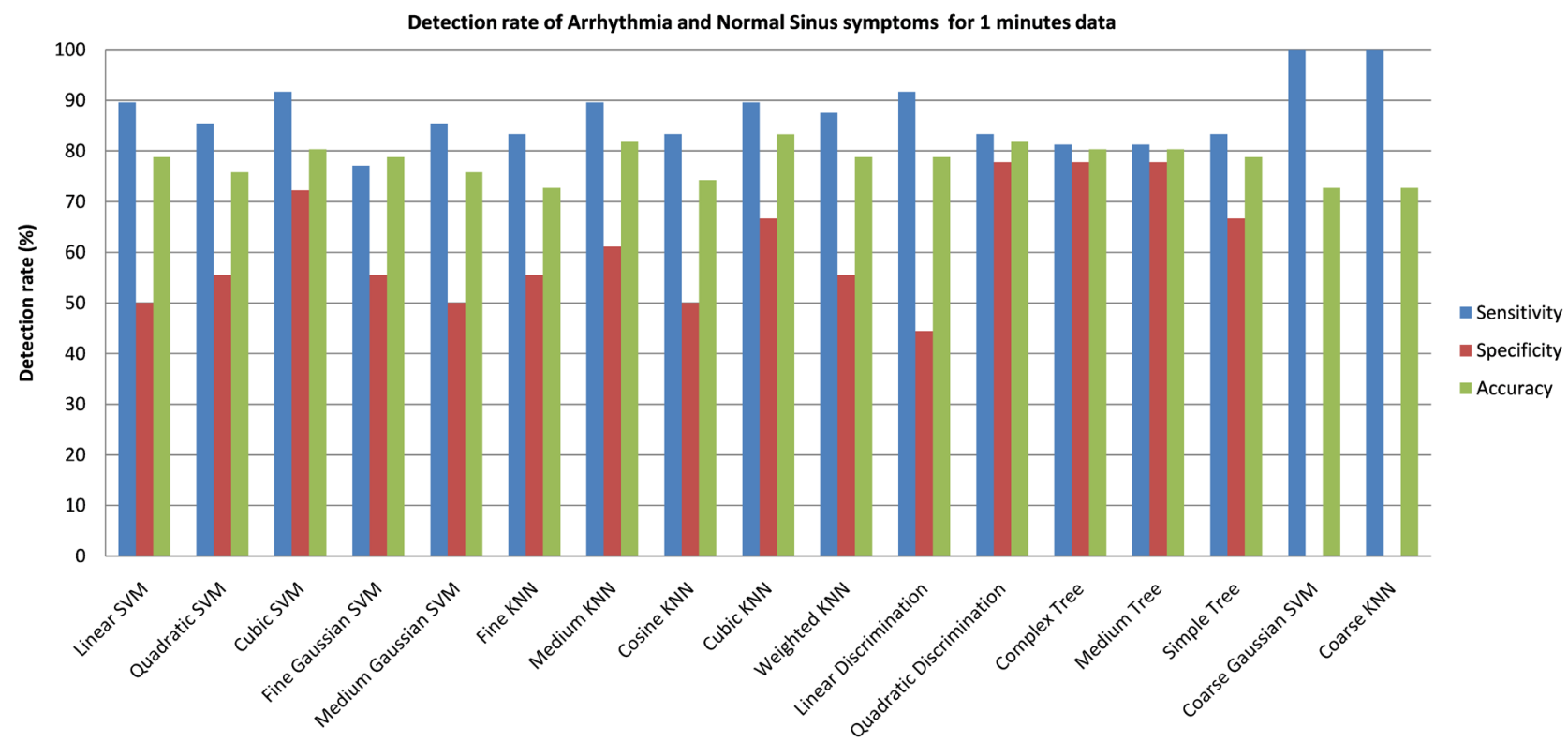

Figure 11. Sensitivity, specificity and accuracy detection rate of arrhythmia and normal sinus symptoms for 1 minute's duration based on 17 various type of classifier.

Table 4. Performance comparison using 17 types of classifiers using the same dataset with 12 hours duration.

\begin{tabular}{|c|c|c|c|}
\hline \multicolumn{4}{|c|}{ Overall performance of 17 various classifier } \\
\hline Classifier Name Accuracy (\%) & & Sensitivity (\%) & Specificity (\%) \\
\hline Linear SVM & 86.4 & 91.6 & 83.3 \\
\hline Quadratic SVM & 86.4 & 91.6 & 83.3 \\
\hline Cubic SVM & 87.9 & 89.5 & 83.3 \\
\hline Fine Gaussian SVM & 87.9 & 97.9 & 61.1 \\
\hline Medium Gaussian SVM & 86.4 & 91.6 & 83.3 \\
\hline Coarse Gaussian SVM & 72.7 & 100 & 0 \\
\hline Fine KNN (Proposed method) & 95.5 & 97.9 & 88.8 \\
\hline Medium KNN & 86.4 & 93.7 & 77.7 \\
\hline Coarse KNN & 72.7 & 100 & 0 \\
\hline Cosine KNN & 68.2 & 83.3 & 33.3 \\
\hline Cubic KNN & 86.4 & 93.7 & 77.7 \\
\hline Weighted KNN & 95.5 & 97.9 & 88.8 \\
\hline Linear Discrimination & 87.9 & 93.7 & 83.3 \\
\hline Quadratic Discrimination & 83.3 & 87.5 & 83.3 \\
\hline Complex Tree & 89.4 & 95.8 & 72.2 \\
\hline Medium Tree & 89.4 & 95.8 & 72.2 \\
\hline Simple Tree & 89.4 & 95.8 & 72.2 \\
\hline
\end{tabular}




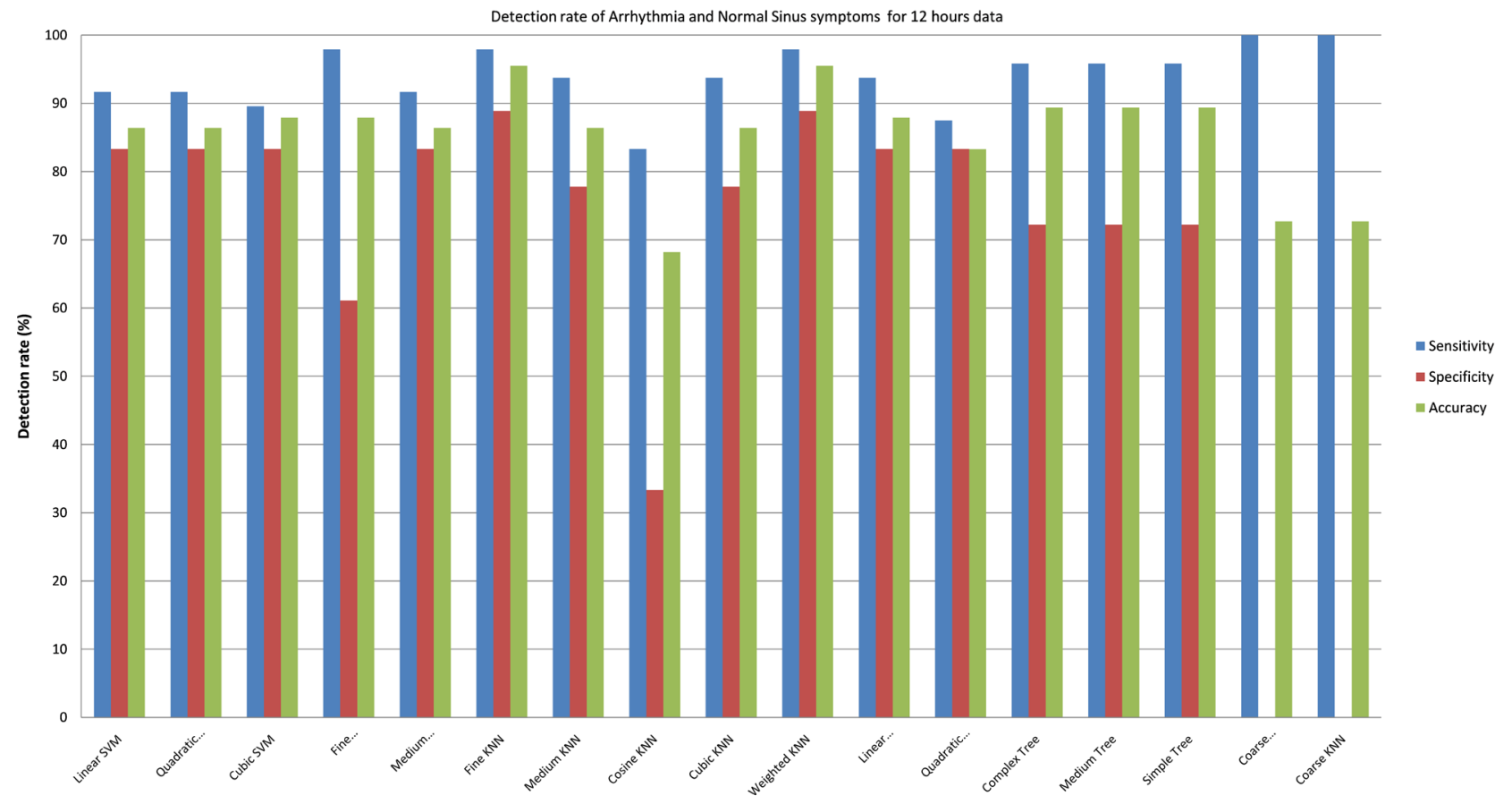

Figure 12. Sensitivity, specificity and accuracy detection rate of arrhythmia and normal sinus symptoms for 12 hours duration based on 17 various type of classifier.

\subsection{Discussion}

To complete this study, an analytical result and discussion was conducted. Based on the result with 12 hours duration data, the distributions pattern of the first periodic slope peak value with the time length are very identical for both symptoms. Correlation coefficient peak value of the first periodic slope in Arrhythmia shows higher score tendency compared to Normal Sinus. Although correlation is conventionally being interpret based on the score size of the relationship, but it is very important to understand the influential factor affecting the size of the score before interpreting it. First, the nature of raw data itself and second is the implementation mechanism of autocorrelation method towards the data. Based on related finding have shown that there are 6 factors that can influence the correlation coefficient score [23]. For this study, there are three factors are correlated with the finding especially on why autocorrelation score for the first periodic slope are much higher with Arrhythmia compared to Normal Sinus. Those factors are amount of variability in the dataset [23] [24], the size of the sample [23] [24] [25] and the characteristic of the sample data itself [23] [24] [25] [26].

Typically, Normal Sinus and Arrhythmia are difference in shape and time. Normal Sinus conditions are more stable and consistent. In Arrhythmia, the symptoms are irregular heartbeat in time series. The heart may beat too fast, too slowly, too early, or irregularly. It was assumed that the amounts of variability in Normal Sinus are much lower compared to Arrhythmia. Although Normal Sinus data may include some noise due to unexpected patient behavior or miss conducted procedure during ECG recording but those events are small compared to 
the nature of Arrhythmia symptom itself. Hence, the presence of an outlier in a dataset can only influencing the correlation score based on the two factors [23]. First, the location of the outlier itself and second, the size of the sample is small enough. In this experiment, each record consists of approximately more than 700,000 data point. Even if there is a small group of outliers included in Normal Sinus data, the overall autocorrelation score will not be affected by it. In this study, it is confirmed that the time length of the data is considered as the biggest influential factor towards the overall classification performance. Due to fact that Arrhythmia randomly appear in time series domain, longer duration is required to accurately detect the disease. In summary, the longer the ECG data is used, the more explicit the behavior of the data can be seen. For that, high accuracy of detecting Arrhythmia can be achieved.

To compare the discrimination performance of the proposed method, Table 5 shows the result of sensitivity and specificity of related studies with the same focus with this research using the same database. Most of the related works are focusing on specific segment in ECG data to classify the symptom. Without relying on any specific feature like the proposed method, the possibilities to discriminate Arrhythmia from Normal Sinus can be done accurately. Best of our knowledge, there is no existing work has proposed as simple method as this study to detect and classify arrhythmia with high accuracy. It is revealed that the proposed mechanism has overcome the other studies in term of simplicity of the mechanism to identify Arrhythmia and abnormalities symptoms regardless the origin of the symptom, and the proposed approach to classify the two symptoms with high accuracy.

Table 5. Overall performance evaluation result comparing this research with other studies.

\begin{tabular}{|c|c|c|}
\hline \multicolumn{3}{|c|}{ Ventricular Arrhythmia Analysis } \\
\hline Method & Sensitivity (\%) & Specificity (\%) \\
\hline Parham et al. [27] & 99.5 & 99.66 \\
\hline Yun-Chi et al. [16] & 98.28 & - \\
\hline Shing et al. [28] & 98.75 & 99 \\
\hline Yun-Chi Yeh et al. [29] & 98.28 & - \\
\hline Joo S. Lim [30] & 99.02 & 96.67 \\
\hline Uday Maji et al. [12] & 99.3 & 99.2 \\
\hline \multicolumn{3}{|c|}{ Atrial Arrhythmia Analysis } \\
\hline Jinseok Lee et al. [31] & 94.7 & 94.4 \\
\hline Uday Maji et al. [12] & 99.8 & 98.4 \\
\hline \multicolumn{3}{|c|}{ Arrhythmia Analysis at Both Segment } \\
\hline Proposed method & 97.9 & 88.8 \\
\hline
\end{tabular}




\section{Conclusion}

In this paper, a novel approach, which statistically detects abnormalities of heart condition based on Arrhythmia symptoms using autocorrelation functions and KNN classifier, was proposed. A variability analysis based on periodic cycle in autocorrelation result was done. It is based on two parameters at first periodic slope of autocorrelation result. In order to discriminate the two symptoms, KNN classifier was used. The effectiveness of the proposed method was evaluated based on 3 performance evaluations metric which are accuracy, sensitivity and specificity. From the result, the overall accuracy was $95.5 \%$, sensitivity of detecting Arrhythmia was $97.5 \%$ and the specificity of detecting Normal Sinus was $88.8 \%$. The comparison approach between this research and other studies shows that the proposed mechanism is robust, flexible and less complexity in detecting abnormalities symptoms like Arrhythmia. In this study, 17 different types of supervised machine learning classifier were used to compare with the proposed classifier. It is proven that fine $\mathrm{KNN}$ has outperformed other classifiers for 12 hours duration segment. 14 types of symptoms had cover in this study and there is no dependency towards any specific characteristic and feature segment in ECG data to identify each Arrhythmia symptom. It is justified the robustness of the proposed method in discriminating abnormalities of heart condition. It is confirmed based on this study that the time length of data is considering the biggest influential factor towards overall classification performance. Therefore, the longer time duration of ECG data is used, the higher accuracy the classifier can be achieved. It is concluded that this research finding can contribute to the medical field to identify Arrhythmia symptom with less complexity procedure in long hour duration.

\section{Conflicts of Interest}

The authors declare no conflicts of interest regarding the publication of this paper.

\section{References}

[1] Ettinger, S.J. (2018) Cardiac Arrhythmias-Diagnosis and Treatment. Cardiology. https://www.vin.com/apputil/content/defaultadv1.aspx?id=3852129\&pid=11181\&\& print $=1$

[2] Lannoy, G.D., Francois, D., Delbeke, J. and Verleysen, M. (2012) Weighted Conditional Random Fields for Supervised Interpatient Heartbeat Classification. IEEE Transactions on Biomedical Engineering, 59, 241-247. https://doi.org/10.1109/TBME.2011.2171037

[3] Osowski, S., Hoai, L. and Markiewicz, T. (2004) Support Vector Machine-Based Expert System for Reliable Heartbeat Recognition. IEEE Transactions on Biomedical Engineering, 51, 582-589. https://doi.org/10.1109/TBME.2004.824138

[4] Dechazal, P., Odwyer, M. and Reilly, R. (2004) Automatic Classification of Heartbeats Using ECG Morphology and Heartbeat Interval Features. IEEE Transactions on Biomedical Engineering, 51, 1196-1206. https://doi.org/10.1109/TBME.2004.827359 
[5] Oliveira, L.S.C.D., Andreao, R.V. and Sarcinelli-Filho, M. (2011) Premature Ventricular Beat Classification Using a Dynamic Bayesian Network. 2011 Annual International Conference of the IEEE Engineering in Medicine and Biology Society, Boston, 30 August-3 September 2011.

[6] Zeng, X.D., Chao, S. and Wong, F. (2011) Ensemble Learning on Heartbeat Type Classification. Proceedings 2011 International Conference on System Science and Engineering, Macao, 8-10 June 2011.

[7] Ince, T., Kiranyaz, S. and Gabbouj, M. (2009) A Generic and Robust System for Automated Patient-Specific Classification of ECG Signals. IEEE Transactions on Biomedical Engineering, 56, 1415-1426. https://doi.org/10.1109/TBME.2009.2013934

[8] Jiang, X., Zhang, L., Zhao, Q. and Albayrak, S. (2006) ECG Arrhythmias Recognition System Based on Independent Component Analysis Feature Extraction. 2006 IEEE Region 10 Conference, Hong Kong, 14-17 November 2006.

[9] Yang, S. and Shen, H. (2013) Heartbeat Classification Using Discrete Wavelet Transform and Kernel Principal Component Analysis. IEEE 2013 Tencon-Spring, Sydney, 17-19 April 2013.

[10] Ye, C., Kumar, B.V.K.V. and Coimbra, M.T. (2012) Heartbeat Classification Using Morphological and Dynamic Features of ECG Signals. IEEE Transactions on Biomedical Engineering, 59, 2930-2941. https://doi.org/10.1109/TBME.2012.2213253

[11] Jiang, W. and Kong, G.S. (2007) Block-Based Neural Networks for Personalized ECG Signal Classification. IEEE Transactions on Neural Networks, 18, 1750-1761. https://doi.org/10.1109/TNN.2007.900239

[12] Maji, U., Mitra, M. and Pal, S. (2017) Characterization of Cardiac Arrhythmias by Variational Mode Decomposition Technique. Biocybernetics and Biomedical Engineering, 37, 578-589. https://doi.org/10.1016/j.bbe.2017.04.007

[13] Giraldo, B., Laguna, P., Jane, R. and Caminal, P. (1995) Automatic Detection of Atrial Fibrillation and Flutter Using the Differentiated ECG Signal. Computers in Cardiology 1995, Vienna, 10-13 September 1995.

[14] Hoppe, B.L., Kahn, A.M., Feld, G.K., Hassankhani, A. and Narayan, S.M. (2005) Separating Atrial Flutter From Atrial Fibrillation with Apparent Electrocardiographic Organization Using Dominant and Narrow F-Wave Spectra. Journal of the American College of Cardiology, 46, 2079-2087. https://doi.org/10.1016/j.jacc.2005.08.048

[15] Christov, I., Bortolan, G. and Daskalov, I. (2001) Sequential Analysis for Automatic Detection of Atrial Fibrillation and Flutter. Computers in Cardiology, Rotterdam, 23-26 September 2001. https://doi.org/10.1109/CIC.2001.977650

[16] Yeh, Y.-C. and Lin, H.-J. (2010) Cardiac Arrhythmia Diagnosis Method Using Fuzzy C-Means Algorithm on ECG Signals. 2010 International Symposium on Computer, Communication, Control and Automation (3CA), Tainan, 5-7 May 2010, 272-275. https://doi.org/10.1109/3CA.2010.5533831

[17] Uchida, S., Mori, A., Kurazume, R., Taniguchi, R.-I. and Hasegawa, T. (2007) Logical DP Matching for Detecting Similar Subsequence. Computer Vision-ACCV 2007: 8th Asian Conference on Computer Vision, Tokyo, 18-22 November 2007, 628-637.

[18] Rajagopal, R. and Ranganathan, V. (2017) Evaluation of Effect of Unsupervised Dimensionality Reduction Techniques on Automated Arrhythmia Classification. Biomedical Signal Processing and Control, 34, 1-8. https://doi.org/10.1016/j.bspc.2016.12.017 
[19] Shen, C.-P., Kao, W.-C., Yang, Y.-Y., Hsu, M.-C., Wu, Y.-T. and Lai, F. (2012) Detection of Cardiac Arrhythmia in Electrocardiograms Using Adaptive Feature Extraction and Modified Support Vector Machines. Expert Systems with Applications, 39, 7845-7852. https://doi.org/10.1016/j.eswa.2012.01.093

[20] PhysioNet (2018) Detrended Fluctuation Analysis (DFA). http://www.physionet.org/

[21] Vijaya, G., Kumar, V. and Verma, H.K. (1998) ANN-Based QRS-Complex Analysis of ECG. Journal of Medical Engineering \& Technology, 22, 160-167. https://doi.org/10.3109/03091909809032534

[22] Florkowski, C.M. (2008) Sensitivity, Specificity, Receiver-Operating Characteristic (ROC) Curves and Likelihood Ratios: Communicating the Performance of Diagnostic Tests. The Clinical Biochemist Reviews, 29, S83-S87.

[23] Goodwin, L.D. and Leech, N.L. (2006) Understanding Correlation: Factors That Affect the Size of $r$. The Journal of Experimental Education, 74, 249-266. https://doi.org/10.3200/JEXE.74.3.249-266

[24] Glass, G.V. and Hopkins, K.D. (2008) Statistical Methods in Education and Psychology. Allyn \& Bacon, Boston.

[25] Lockhart, R.S. (1998) Introduction to Statistics and Data Analysis for the Behavioral Sciences. W.H. Freeman, New York.

[26] Sprinthall, R.C. (2014) Basic Statistical Analysis. Pearson, London.

[27] Ghorbanian, P., Ghaffari, A., Jalali, A. and Nataraj, C. (2010) Heart Arrhythmia Detection Using Continuous Wavelet Transform and Principal Component Analysis with Neural Network Classifier. Computing in Cardiology, Belfast, 26-29 September 2010, 669-672.

[28] Pan, S.-T., Chiou, Y.-J., Hong, T.-P. and Chen, H.-C. (2013) Automatic Recognition for Arrhythmias with the Assistance of Hidden Markov Model. 2013 9th International Conference on Information, Communications \& Signal Processing, Tainan, 10-13 December 2013, 1-5.

[29] Yeh, Y.-C. (2009) An Analysis of ECG Beats by Using the Mahalanobis Distance Method. 2009 Fourth International Conference on Innovative Computing, Information and Control (ICICIC), Kaohsiung, 7-9 December 2009, 1460-1463. https://doi.org/10.1109/ICICIC.2009.75

[30] Shyu, L.-Y., Wu, Y.-H. and Hu, W. (2004) Using Wavelet Transform and Fuzzy Neural Network for VPC Detection from the Holter ECG. IEEE Transactions on Biomedical Engineering, 51, 1269-1273. https://doi.org/10.1109/TBME.2004.824131

[31] Lee, J., Reyes, B.A., Mcmanus, D.D., Maitas, O. and Chon, K.H. (2013) Atrial Fibrillation Detection Using an iPhone 4S. IEEE Transactions on Biomedical Engineering, 60, 203-206. https://doi.org/10.1109/TBME.2012.2208112 